\title{
Influences of general self-efficacy and weight bias internalization on physical activity in
}

\section{bariatric surgery candidates}

Claudia Hübner, M.Sc., ${ }^{\text {a,b }}$ Sabrina Baldofski, M.Sc., ${ }^{\text {a,b }}$ Markus Zenger, Ph.D., ${ }^{b}$ Wolfgang Tigges, M.D., ${ }^{\mathrm{c}}$ Beate Herbig, M.D., ${ }^{\mathrm{d}}$ Christian Jurowich, M.D., ${ }^{\mathrm{e}}$ Stefan Kaiser, M.D., ${ }^{\mathrm{f}}$ Arne Dietrich, M.D., ${ }^{\mathrm{a}}$ \& Anja Hilbert, Ph.D. ${ }^{\mathrm{a}, \mathrm{b}}$

- Original Article -

The final publication is available at http://dx.doi.org/10.1016/j.soard.2014.11.013. (C) 2015. This manuscript version is made available under the CC-BY-NC-ND 4.0 license [Link to: http://creativecommons.org/licenses/by-nc-nd/4.0/]

\section{Affiliation:}

a Leipzig University Medical Center, Integrated Research and Treatment Center AdiposityDiseases, Leipzig, Germany

${ }^{\mathrm{b}}$ Leipzig University Medical Center, Department for Medical Psychology and Medical Sociology, Leipzig, Germany

c Department of General Surgery, Asklepios Clinic, Hamburg, Germany

d Schön Klinik Hamburg Eilbek Bariatric Clinic, Hamburg, Germany

e Department of General Surgery, University Hospital Würzburg, Würzburg, Germany

${ }^{\mathrm{f}}$ Department of Visceral, Pediatric, and Vascular Surgery, Hospital Konstanz, Konstanz, Germany

\section{Correspondence to:}


Claudia Hübner, M.Sc., Leipzig University Medical Center, Integrated Research and Treatment Center AdiposityDiseases, Behavioral Medicine, Philipp-Rosenthal-Strasse 27, 04103 Leipzig, Germany

E-Mail: claudia.huebner@medizin.uni-leipzig.de, phone: +49-341-97-15380, fax: +49-34197-15359

\section{Conflicts of interest statement:}

The authors declare no conflicts of interest with respect to the content of this manuscript.

\section{Acknowledgements:}

The authors' responsibilities were as follows - SB, WT, BH, CJ, SK, AD, and AH developed the protocol and contributed to data collection, and $\mathrm{CH}$ as well as $\mathrm{MZ}$ analyzed the data. All authors were involved in writing the manuscript and had approval of the submitted and published versions.

This work was supported by the Federal Ministry of Education and Research (BMBF), Germany, FKZ: 01EO1001.

The authors thank Christine Stroh, M.D., Kathrin Hohl, Heike Laser, Martina Pabst, M.D., Peter Pick, M.D., Johannes Sander, M.D., and Yvonne Sockolowsky for data collection at the study centers, and Almut Rudolph, Ph.D., and Carolyn Edwards, B.Sc., for editing the manuscript. 


\section{Abstract}

Background: Physical activity (PA) seems to be important for long-term weight loss following bariatric surgery, however, studies provide evidence for insufficient PA levels in bariatric patients. Research found self-efficacy to be associated with PA and weight bias internalization, whose influence on mental and physical health has been shown in recent studies.

Objectives: The purpose of the present study was to investigate the influence of general self-efficacy on PA, mediated by weight bias internalization.

Setting: Consecutive multicenter registry study conducted in six German bariatric surgery centers.

Methods: In $N=179$ bariatric surgery candidates, general self-efficacy, weight bias internalization, and different intensities of PA were assessed by self-report questionnaires. Structural equation modeling was used to analyze the assumed mediational relationship.

Results: After controlling for sociodemographic variables, weight bias internalization fully mediated the association between general self-efficacy and moderate-intense as well as vigorous-intense PA. Lower general self-efficacy predicted greater weight bias internalization, which in turn predicted lower levels of moderate-intense and vigorous-intense PA.

Conclusions: The results suggest an influence of weight bias internalization on preoperative PA in bariatric surgery candidates. Subsequently, implementation of interventions addressing weight bias internalization in the usual treatment of bariatric surgery candidates might enhance patients’ preoperative PA, while longitudinal analyses are needed in order to further examine its predictive value on PA following bariatric surgery.

Keywords: weight bias internalization; self-efficacy; physical activity; mediation. 


\section{Introduction}

Physical activity (PA) might be essential for long-term weight loss following bariatric surgery, yet previous studies yielded insufficient pre- and postoperative activity levels in bariatric patients. Based on findings demonstrating associations between self-efficacy and PA, and the influence of weight bias internalization (WBI) on physical and mental health, the present study aimed to analyze the mediating role of WBI on the relationship between general self-efficacy and preoperative PA.

Bariatric surgery is considered the most effective treatment of extreme obesity at body mass index $(\mathrm{BMI}) \geq 40 \mathrm{~kg} / \mathrm{m}^{2(1)}$, however, postoperative outcomes depend tremendously on patients' behavior modification ${ }^{(2)}$. There is growing evidence that postoperative PA might be crucial for bariatric surgery patients to achieve long-term weight loss ${ }^{(2)}$. However, recent studies in bariatric surgery patients revealed low levels of pre- and postoperative PA compared to general health recommendations ${ }^{(3)}$. Preoperative PA might decrease postoperative complications ${ }^{(4)}$ and predicted postoperative $\mathrm{PA}^{(5)}$, hence, psychosocial determinants of PA should be examined preoperatively and might be addressed by potential interventions.

Reviewing previous research, self-efficacy was suggested predominantly as a psychosocial determinant of $\mathrm{PA}^{(6)}$. Self-efficacy is defined as self-beliefs of accomplishing a behavior due to personal capabilities ${ }^{(7)}$ and can be divided into global self-beliefs (general self-efficacy) or self-beliefs regarding different situations or behaviors (domain-specific selfefficacy, e.g., PA specific self-efficacy $)^{(8)}$. Numerous studies provide evidence for a positive correlation between general as well as domain-specific self-efficacy and PA in various nonweight-specified samples ${ }^{(9)}$. This positive association with general self-efficacy was found in obese individuals as well ${ }^{(10)}$, but the relationship has not yet been investigated in bariatric 
patients, as a specific subsample differing not only in weight status. After adjusting for BMI, populations differed in diverse psychosocial aspects, with bariatric patients reporting less favorable values than obese ones (e.g., in physical and social functioning, vitality) ${ }^{(11)}$. Further studies established the predictive value of PA specific self-efficacy on levels of PA in nonweight-specified samples ${ }^{(12)}$, but interpretation of these findings with regard to general selfefficacy is limited.

Further, the concept of WBI has gained growing interest in research on health impairments in overweight and obesity. WBI is characterized by applying predominant negative weight stereotypes and prejudices to the own person ${ }^{(13)}$. It emerged as an important factor in overweight and obese individuals as previous studies yielded associations with higher levels of psychopathology ${ }^{(13)}$ and poorer physical and mental health ${ }^{(14)}$. In addition, a recently published study observed a negative relationship between WBI and PA in overweight and obese women ${ }^{(15)}$, but the generalizability of these results to bariatric surgery candidates has not yet been investigated. Regarding the relationship between self-efficacy and WBI in overweight and obese individuals, WBI was negatively associated with PA specific self-efficacy ${ }^{(15)}$ and with core self-evaluation, including self-efficacy, self-esteem, neuroticism, and locus of control ${ }^{(16)}$.

In this context, the present study is the first to investigate the association between WBI and PA, as well as the role of WBI as a mediator of the relationship between selfefficacy and PA in bariatric surgery candidates. We decided in favor of general self-efficacy because, as a global personality disposition, it allows for higher generalizability than its domain specific counterpart. Thus, we aimed to examine the impact of preoperative general self-efficacy and WBI on walking, moderate-intense, and vigorous-intense PA in bariatric surgery candidates. Referring to previous findings, we hypothesized that lower general selfefficacy would lead to higher WBI, which in turn would lead to decreased PA. 


\section{Materials and Methods}

\section{Participants}

This study was part of the Psychosocial Registry for Bariatric Surgery (PRAC study), which longitudinally assesses psychosocial parameters in a consecutive sample of bariatric surgery patients in six German bariatric surgery centers. The PRAC study was approved by the authorized ethics committee of each study center and is described in detail elsewhere ${ }^{(17)}$. This study included all preoperative data of $N=179$ extremely obese bariatric surgery candidates, all of whom provided written informed consent prior to study participation. Data collection proceeded independently of clinical treatment and patients were informed that the information obtained would not be shared with the surgical team.

As summarized in Table 1, the sample consisted of 121 women (67.6\%) and 58 men (32.4\%) with a mean age of $M=46.58$ years $(S D=10.43)$ and a mean BMI of $M=48.73$ $\mathrm{kg} / \mathrm{m}^{2}(S D=7.34)$. While class III obesity $\left(\mathrm{BMI} \geq 40.0 \mathrm{~kg} / \mathrm{m}^{2}\right)$ was present in the majority of patients ( $n=167 ; 93.30 \%)$, class II obesity was diagnosed in only 12 patients $(6.70 \%)$.

Measures

Predictor variable. The General Self-Efficacy Scale (GSE) ${ }^{(18)}$ was used to measure global self-beliefs of coping with demanding situations due to personal competence ${ }^{(8)}$. It consists of 10 items (e.g., "I can always manage to solve difficult problems if I try hard enough”) rated on a 4-point Likert scale ranging from $1=$ not at all true to $4=$ exactly true. A sum score was computed with higher scores indicating greater general self-efficacy. Good validity and adequate reliability have been shown in various studies ${ }^{(8)}$. Internal consistency in this study's sample was $\alpha=.94$. 
Mediator variable. The Weight Bias Internalization Scale (WBIS) ${ }^{(13,19)}$ was used to assess internalized weight bias defined as the overweight or obese individual's degree of approval to statements applying predominant public weight stigma to the own person. Each of the 11 items (e.g., “Because of my weight, I don’t understand how anyone attractive would want to date me”) was rated on a 7-point Likert scale from $0=$ strongly disagree to $7=$ strongly agree. As recent psychometric analyses of the WBIS recommended removal of item 1 due to insufficient item-total correlations ${ }^{(19)}$, WBIS mean scores were computed of the remaining 10 items, with higher scores indicating greater WBI. The WBIS has shown good validity and excellent reliability ${ }^{(19)}$. Internal consistency in the present study sample was $\alpha=$ 86 .

Outcome variable. To assess participant's amount of PA, we used the selfadministered 9-item short-form of the International Physical Activity Questionnaire (IPAQ$\mathrm{SF})^{(20)}$. Participants rated how much time they spent on walking, moderate-intense (e.g., regular bicycling, light lifting), vigorous-intense (e.g., fast bicycling, heavy lifting), and sedentary activities over the last seven days. To assess only PA in the present study, we excluded the item assessing sedentary activities from all analyses. A sum score in minutes per week was computed for each subscale. The IPAQ-SF was originally designed for assessment in population studies ${ }^{(20)}$, however, it is a widely administered self-report questionnaire in various studies on PA with small to medium sample sizes ${ }^{(9,21)}$.

\section{Data Analytic Plan}

The distributions of the IPAQ-SF subscales were negatively skewed with more frequent low than high amounts of PA, thus, we transformed the sum scores by extracting the square roots to approximately normalize the distributions ${ }^{(22)}$. 
Regarding the postulated model, the structural equation modeling approach was used and statistical analyses were carried out with AMOS'м 20 using the following model fit indices: $\chi^{2}$ test statistic; the minimum discrepancy, divided by its degrees of freedom (CMIN/DF); the goodness-of-fit-index (GFI); the comparative-fit index (CFI); the TuckerLewis-Index (TLI); and the root mean square error of approximation (RMSEA). A nonsignificant $\chi^{2}$ value indicates that the model is compatible with the data ${ }^{(23)}$. For a good model fit, the ratio CMIN/DF should be close to 2, and GFI, CFI as well as TLI should be $>0.95$ for a good or $>.90$ for an acceptable model fit ${ }^{(23)}$. Furthermore, RMSEA values $<0.06$ indicate a good and values $<0.10$ an acceptable model fit ${ }^{(24)}$. The model was tested using correlation matrices and it was estimated with the full information maximum likelihood method approach.

In a first step, the direct relationships of general self-efficacy and the proposed outcome variables of PA were tested, while controlling for the sociodemographic variables of age, sex, and education. General self-efficacy emerged as a significant predictor of vigorousintense activities (standardized value: .15, $p<.05$ ) and moderate-intense activities (standardized value: .17; $p<.05$ ), but not for walking (standardized value: .08; $p=.30$ ). In a second step, WBI was added as a mediator of the relationship between general self-efficacy and PA outcome variables. Testing the influence of the sociodemographic variables in both steps, these variables were allowed to predict every other manifest variable in the model. Subsequently, all nonsignificant relations were deleted and significant relationships remained in the model.

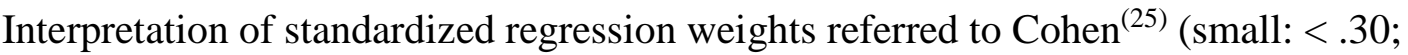
medium: $\geq .30$ and $<.50$; large: $\geq .50$ ). A two-tailed $\alpha$ of .05 was applied for all statistical tests. 


\section{Results}

As outlined in Table 1, the sample reported a mean GSE score of $M=30.05$ (SD = 6.06) and a mean WBIS score of $M=4.95(S D=1.19)$. On average, participants walked $M=$ 607.98 minutes ( $S D=768.47$ minutes) during the last week. They further engaged in $M=$ 291.02 minutes $(S D=635.87$ minutes $)$ of moderate-intense and $M=380.90$ minutes $(S D=$ 732.35 minutes) of vigorous-intense PA.

The structural equation model presented in Figure 1 depicts all significant paths of the tested model. After controlling for sociodemographic variables (age, sex, and education), lower general self-efficacy predicted greater WBI (medium effect), which in turn predicted a smaller amount of walking, moderate-intense PA, and vigorous-intense PA (small effects). Regarding the effects of sociodemographic variables, a higher age was significantly associated with less vigorous-intense PA, while general self-efficacy yielded an effect of sex, with females showing lower scores than males (small effects). However, education was not significantly associated with any of the integrated variables (and therefore was not depicted in the model). Based on the $\chi^{2}$ test statistic, the model fit the data: $\chi^{2}(12)=20.707, p=.06$. Further, a good or at least an acceptable model fit is indicated by the following structural equation modeling fit indices: $\mathrm{CMIN} / \mathrm{DF}=1.723 ; \mathrm{GFI}=.968 ; \mathrm{CFI}=.942 ; \mathrm{TLI}=.898$; RMSEA $=.064$.

As general self-efficacy did not predict walking in the first model without mediator, we only found a full mediation effect of WBI on moderate-intense and vigorous-intense PA: when WBI was included, general self-efficacy did not add to the prediction of these two aspects of PA. 


\section{Discussion}

In the present study unique in examining this issue in bariatric surgery candidates, we found that WBI fully mediated the association between general self-efficacy and moderateintense as well as vigorous-intense preoperative PA: lower general self-efficacy predicted greater WBI, which in turn predicted a smaller amount of these aspects of PA.

Our results replicate findings regarding the association between general self-efficacy and PA levels ${ }^{(12)}$, as well as the association between self-efficacy and WBI, in bariatric surgery candidates ${ }^{(15,16)}$. They further provide evidence on the negative relationship between WBI and PA that has been found in overweight and obese females, but has not yet been investigated in bariatric surgery candidates ${ }^{(15)}$. Beyond this, the complete mediational effect of WBI indicates that general self-efficacy does not contribute supplementally to the prediction of moderate-intense and vigorous-intense PA when WBI is included. However, there was no mediational effect of WBI on the relationship between general self-efficacy and walking due to the missing association in the model without mediator. A possible explanation might be that, irrespective of their general self-efficacy, patients walk for everyday transportation rather than for exercising. With regard to sociodemographic variables, we replicated findings of sex-specific general self-efficacy ${ }^{(8)}$ and age-related vigorous $\mathrm{PA}^{(26)}$.

Altogether, WBI emerged as an influencing factor of preoperative PA in bariatric surgery candidates. This finding is essential because preoperative PA might decrease postoperative complications $^{(4)}$ and was found to be a predictor of postoperative PA indicating that PA barriers may persist ${ }^{(5)}$. In this context, our results underline the importance of further studies prospectively investigating the predictive value of WBI.

Our findings need to be interpreted while taking into account the strengths and limitations of this study. Strengths include the multicenter design as well as the large sample size of bariatric surgery candidates. Further, internationally well-established and widely 
accepted self-report questionnaires were applied. Moreover, socially desired ratings of patients were reduced because data collection was unrelated to clinical procedures and patients were informed that the information obtained would not be shared with the surgical team.

As a restriction, use of the IPAQ-SF was recommended primarily for national prevalence studies $^{(20)}$. While we did not investigate a representative sample of the population, our clinical sample of bariatric surgery candidates can be classified as large, justifying the use of this instrument. Another limitation is the subjective PA assessment as various studies observed an overestimation of PA in self-report questionnaires compared to objective methods suggesting subjective measures to be a less reliable indicator of $\mathrm{PA}^{(27)}$. As the design of this study prevented the use of accelerometers, further studies are needed to replicate these findings with objectively quantified PA. Though, it should be mentioned that accelerometers are suited to capture most, but not all types of PA, e.g., aquatics. In addition, bariatric surgery candidates are a rather homogenous sample with regard to BMI. It might be the purpose of future research to examine the relevance and generalizability of our results to patients in conservative weight loss treatment because increasing overweight and obese individuals’ PA is important in any treatment ${ }^{(28)}$. Comparable to previous research on WBI, interpretation of our results is further limited due to cross-sectional data preventing causal attributions. The postulated model was based on prior findings, nevertheless, it might be possible that there are some associations in the opposite direction, e.g., PA might enhance general self-efficacy. However, prospective monitoring of the present sample following bariatric surgery will be proceeded within the PRAC study. These longitudinal analyses will allow causal attributions between preoperative WBI and postoperative PA as well as weight loss and will help to clarify if WBI should be targeted preoperatively to foster an active lifestyle in the long term. 


\section{Conclusions}

Our results might provide practitioners with information underlining the importance of WBI reduction for enhancing PA in bariatric surgery candidates. Interventions aiming at increasing PA in general frequently target an increase in self-efficacy ${ }^{(29)}$, as it has been shown to be associated with PA in various studies ${ }^{(6,12)}$. However, based on our results, PA behavior modification should focus on WBI rather than self-efficacy, especially in bariatric surgery candidates, for whom increasing PA is challenging ${ }^{(5)}$. While longitudinal studies investigating the role of bariatric surgery candidates' WBI on postoperative PA are needed, our findings emphasize that addressing WBI in patients with less favorable scores might enhance preoperative PA. In this context, administering the $\mathrm{WBIS}^{(13,19)}$ as a screening instrument at the initial visit could be the key in identifying bariatric patients with high levels of WBI in relation to population norms ${ }^{(19)}$. Subsequently, these patients should be considered for preoperative counseling to reduce WBI, i.a., by providing information on weight bias in society, leading the patient to its critical consideration, and detaching self-evaluation from stereotypes $^{(30)}$. However, as there is only limited research on the reduction of WBI, our results highlight the clinical demand for further development and evaluation of existing interventions targeting WBI. Subsequently, advanced interventions might be implemented in future treatment of bariatric surgery candidates with increased WBI to improve preoperative PA counseling and its outcomes. 


\section{References}

1. Maggard MA, Shugarman LA, Suttorp M, et al. Meta-analysis: Surgical treatment of obesity. Ann Intern Med 2005;142:547-59.

2. Evans RK. The role of physical activity participation in weight loss outcomes following weight loss surgery. Am J Lifestyle Med 2010;4:124-29.

3. King WC, Bond DS. The importance of preoperative and postoperative physical activity counseling in bariatric surgery. Exercise Sport Sci R 2013;41:26-35.

4. McCullough PA, Gallagher MJ, Dejong AT, et al. Cardiorespiratory fitness and shortterm complications after bariatric surgery. Chest 2006;130:517-25.

5. King WC, Hsu JY, Belle SH, et al. Pre- to postoperative changes in physical activity: Report from the longitudinal assessment of bariatric surgery-2 (LABS-2). Surg Obes Relat Dis 2012;8:522-32.

6. Bauman AE, Reis RS, Sallis JF, Wells JC, Loos RJF, Martin BW. Correlates of physical activity: why are some people physically active and others not? Lancet 2012;380:258-71.

7. Bandura A. Self-efficacy. The exercise of control. New York, Freeman, 1997, pp 1604.

8. Schwarzer R, Bäßler J, Kwiatek P, Schröder K, Zhang JX. The assessment of optimistic self-beliefs: Comparison of the German, Spanish, and Chinese versions of the General Self-Efficacy Scale. Appl Psychol-Int Rev 1997;46:69-88. 
9. Rutkowski EM, Connelly CD. Self-efficacy and physical activity in adolescent and parent dyads. J Spec Pediatr Nurs 2012;17:51-60.

10. Bonsaksen T, Lerdal A, Fagermoen MS. Factors associated with self-efficacy in persons with chronic illness. Scand J Psychol 2012;53:333-39.

11. van Nunen AMA, Wouters EJM, Vingerhoets AJJM, Hox JJ, Geenen R. The healthrelated quality of life of obese persons seeking or not seeking surgical or non-surgical treatment: A meta-analysis. Obes Surg 2007;17:1357-66.

12. McAuley E, Elavsky S, Motl RW, Konopack JF, Hu L, Marquez DX. Physical activity, self-efficacy, and self-esteem: Longitudinal relationships in older adults. J Gerontol B Psychol Sci Soc Sci 2005;60:P268-75.

13. Durso LE, Latner JD. Understanding self-directed stigma: Development of the Weight Bias Internalization Scale. Obesity 2008;16:80-86.

14. Latner JD, Durso LE, Mond JM. Health and health-related quality of life among treatment-seeking overweight and obese adults: associations with internalized weight bias. J Eat Disord 2013;1:1-6.

15. Pearl RL, Puhl RM, Dovidio JF. Differential effects of weight bias experiences and internalization on exercise among women with overweight and obesity. J Health Psychol Epub 2014 Feb 6.

16. Hilbert A, Braehler E, Haeuser W, Zenger M. Weight bias internalization, core selfevaluation, and health in overweight and obese persons. Obesity 2014;22:79-85.

17. Baldofski S, Tigges W, Herbig B, et al. Non-normative eating behavior and psychopathology in prebariatric patients with binge-eating disorder and night eating syndrome. Surg Obes Relat Dis. Forthcoming 2014. 
18. Schwarzer R, Jerusalem M. Generalized Self-Efficacy Scale. In Weinman J, Wright S, Johnston M (eds). Measures in health psychology: A user’s portfolio. Causal and control beliefs. Windsor, NFER-NELSON, 1995, pp 35-37.

19. Hilbert A, Baldofski S, Zenger M, Löwe B, Kersting A, Braehler E. Weight Bias Internalization Scale: Psychometric properties and population norms. PLoS ONE 2014;9:e86303.

20. Craig CL, Marshall AL, Sjöström M, et al. International Physical Activity Questionnaire: 12-country reliability and validity. Med Sci Sports Exerc 2003;35:138195.

21. Bond DS, Phelan S, Wolfe LG, et al. Becoming physically active after bariatric surgery is associated with improved weight loss and health-related quality of life. Obesity 2009;17:78-83.

22. Tukey JW. Exploratory data analysis. Massachusetts: Addison-Wesley, Reading; 1977.

23. Schermelleh-Engel K, Moosbrugger H, Müller H. Evaluating the fit of structural equation models: Tests of significance and descriptive goodness-of-fit Measures. Meth Psychol Res 2003;8:23-74.

24. Hu L, Bentler PM. Cutoff criteria for fit indexes in covariance structure analysis: Conventional criteria versus new alternatives. Struct Equ Modeling 1999;6:1-55.

25. Cohen J. Statistical power analysis for the behavioral sciences, 2nd ed. Hillsdale NJ, Erlbaum, 1988, pp 1-567.

26. Troiano RP, Berrigan D, Dodd KW, Mâsse LC, Tilert T, McDowell M. Physical activity in the United States measured by accelerometer. Med Sci Sports Exerc 2008;40:181-88. 
27. King WC, Belle SH, Eid GM, et al. Physical activity levels of patients undergoing bariatric surgery in the Longitudinal Assessment of Bariatric Surgery study. Surg Obes Relat Dis 2008;4:721-28.

28. Jensen MD, Ryan DH, Apovian CM, et al. 2013 AHA/ACC/TOS Guideline for the management of overweight and obesity in adults: A Report of the American College of Cardiology/American Heart Association Task Force on Practice Guidelines and The Obesity Society. J Am Coll Cardiol 2014;63:2985-3023.

29. Higgins TJ, Middleton KR, Winner L, Janelle CM. Physical activity interventions differentially affect exercise task and barrier self-efficacy: A meta-analysis. Health Psychol 2014;33:891-903.

30. Hilbert A, Tuschen-Caffier B. Essanfälle und Adipositas. Ein Manual zur kognitivbehavioralen Therapie der Binge-Eating-Störung [Binge-eating and obesity: Cognitivebehavioral therapy for binge-eating disorder]. Göttingen, Hogrefe, 2010, pp 1-117. German. 
Table 1. Sample characteristics $(N=179)$.

\begin{tabular}{|c|c|c|}
\hline & $M, n$ & $S D, \%$ \\
\hline Sex, females & 121 & 67.60 \\
\hline Age, years & 46.58 & 10.43 \\
\hline Education, $<12$ years & 141 & $80.57^{1}$ \\
\hline BMI, kg/m² & 48.73 & 7.34 \\
\hline \multicolumn{3}{|l|}{ Weight status } \\
\hline Class II obesity $\left(35.0 \leq \mathrm{BMI} \leq 39.9 \mathrm{~kg} / \mathrm{m}^{2}\right)$ & 12 & 6.70 \\
\hline Class III obesity (BMI $\geq 40.0 \mathrm{~kg} / \mathrm{m}^{2}$ ) & 167 & 93.30 \\
\hline General Self-efficacy (GSE) & 30.05 & 6.06 \\
\hline Weight bias internalization (WBIS) & 4.95 & 1.19 \\
\hline \multicolumn{3}{|l|}{ Physical activity (IPAQ-SF) } \\
\hline Vigorous-intense physical activity, min/week & 380.90 & 732.35 \\
\hline Moderate-intense physical activity, min/week & 291.02 & 635.87 \\
\hline Walking, min/week & 607.98 & 768.47 \\
\hline
\end{tabular}

Notes. BMI, body mass index; GSE, General Self-Efficacy Scale $\left(10^{*}-40\right.$, less favorable scores are asterisked); WBIS, Weight Bias Internalization Scale (1-7*); IPAQ-SF, International Physical Activity Questionnaire - Short Form.

${ }^{1}$ Calculation of \% from valid cases $(n=175)$. 


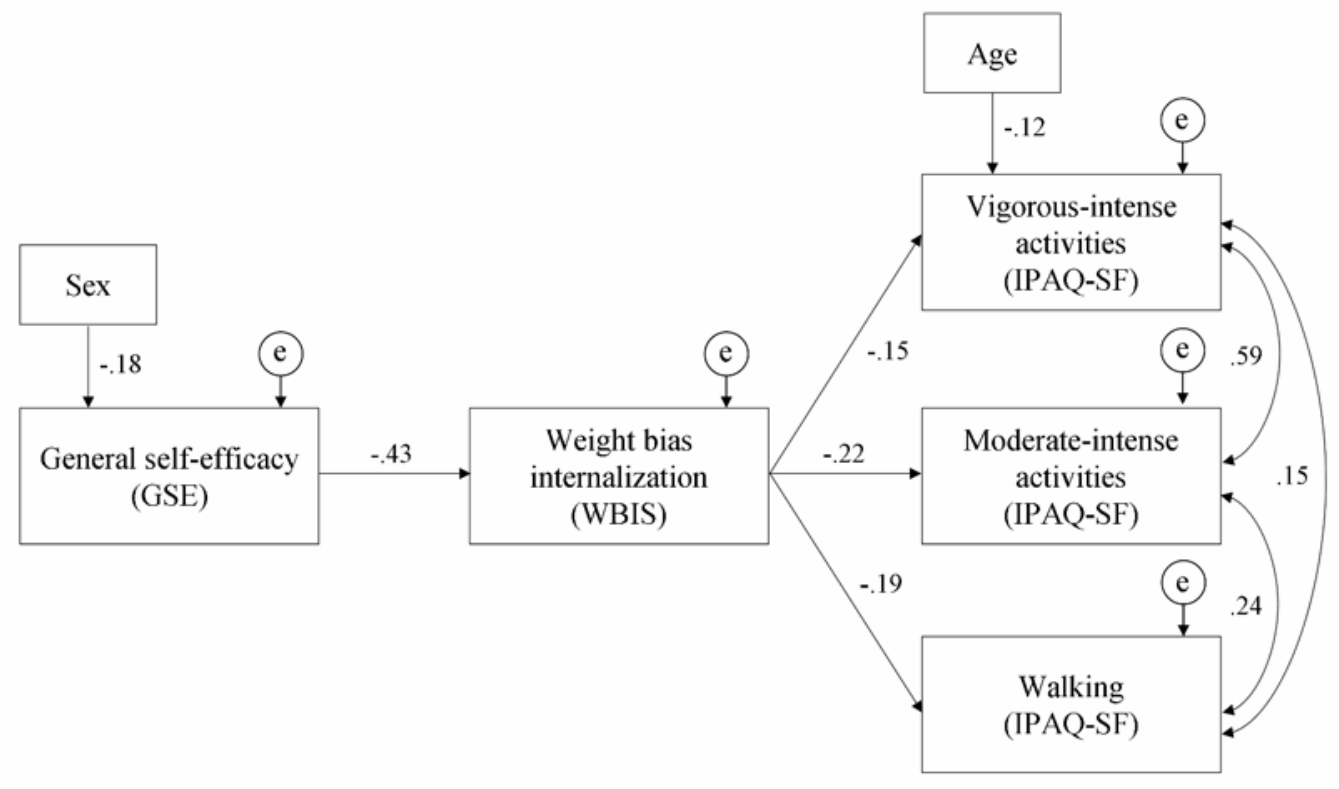

Figure 1. Structural equation model on the mediating role of weight bias internalization on the relationship between general self-efficacy and physical activity.

Notes. Standardized values are depicted $(p<.05)$. GSE, General Self-Efficacy Scale $(10 *-40$, less favorable scores are asterisked); WBIS, Weight Bias Internalization Scale (1-7*); IPAQSF, International Physical Activity Questionnaire - Short Form (min/week). 